\title{
A fast and efficient crosstalk-free algorithm for routing in optical multistage interconnection networks
}

\begin{abstract}
In this paper, a fast and efficient crosstalk-free routing algorithm is proposed to enhance message routing in optical multistage interconnection networks (OMINs). The new Fast ZeroXY algorithm is designed based on the Zero algorithms, which uses the time dilation approach to eliminate the negative effect of crosstalk associated with optical switching in the optical Omega network. To evaluate the performance of the new algorithm, a crosstalk-free version of the original ZeroXY algorithm is developed extended from the Improved ZeroXY algorithm, called the Modified ZeroXY algorithm. The Fast ZeroXY algorithm is shown to efficiently route permutations without crosstalk with improved routing time compared to the original crosstalk- free ZeroXY algorithm.
\end{abstract}

Keyword: Crosstalk-free routing algorithm; Optical crosstalk; Optical multistage interconnection networks 